\title{
Estilos de Aprendizaje de los Estudiantes de la Facultad de Ingeniería de la Universidad de Cartagena (Colombia)
}

\author{
Diofanor Acevedo(1), Shirley Cavadia(2) y Armando Alvis(3) \\ (1) Universidad de Cartagena, Facultad de Ingeniería, Programa de Ingeniería de Alimentos, Avenida el \\ Consulado, Calle 30 No. 48-152. Cartagena, Bolívar-Colombia (e-mail:diofanor3000@gmail.com) \\ (2) Universidad de Cartagena, Facultad de Ciencias Farmacéuticas, Programa de Química Farmacéutica, \\ Zaragocilla, Campus de la Salud, Cartagena, Bolívar-Colombia \\ (3) Universidad de Córdoba, Facultad de Ingeniería, Departamento de Ingeniería de Alimentos, Carrera 6 \\ No 76-103, Km. 3, vía Cereté, Córdoba-Colombia
}

Recibido Sep. 29, 2014; Aceptado Dic. 1, 2014; Versión final recibida Feb. 2, 2015

\begin{abstract}
Resumen
Se analizaron los estilos de aprendizaje en estudiantes de la Facultad de Ingeniería de la Universidad de Cartagena. La muestra consistió de 144 estudiantes, 72 mujeres y 72 hombres de edad promedio 22 años. Se aplicó el cuestionario Honey-Alonso sobre estilos de aprendizaje, para identificar el comportamiento activo, reflexivo, teórico y pragmático de los estudiantes. Para ello, se realizó un análisis de varianza bifactorial y una correlación de Pearson $(p \leq 0,05)$. El estilo predominante fue reflexivo, con $31,9 \%$ de los hombres y $36,1 \%$ de las mujeres en esta categoría. No hubo diferencias significativas por género $(p>0,05)$. En los hombres hubo correlación directa entre el reflexivo y pragmático $(p<0,05)$ y en las mujeres se encontró una relación inversa entre el activo y teórico $(p<0,05)$. Los estudiantes fueron analíticos y prefirieron la aplicación de las ideas. Esto debe tenerse en cuenta por parte de los docentes en los métodos de enseñanza.
\end{abstract}

\section{Learning Styles of Students of the Faculty of Engineering of the University of Cartagena (Colombia)}

\begin{abstract}
Learning styles on students of the Faculty of Engineering of the University of Cartagena were analyzed. The sample consisted of 144 students, 72 women and 72 men of average age 22 years. The Honey-Alonso Questionnaire of Learning Styles was applied to identify the active, reflexive, theorist and pragmatic behavior of the students. For this, analysis of variance and Pearson correlation $(p \leq 0,05)$ was performed. The predominant style was reflective, with $31,9 \%$ of men and $36,1 \%$ of women, and averages of 12,9 and 12,4 respectively. There were no significant differences by gender $(p>0,05)$. The general preference was moderate $(33,3 \%)$. In men, there was a direct correlation between reflective and pragmatic $(p<0,05)$ and in women and inverse relation between active and theorist $(p<0,05)$. Students show a more analytical approach and preferred the implementation of ideas. This must be considered by instructors in their teaching methods.
\end{abstract}

Keywords: Honey-Alonso questionnaire, learning styles, engineering teaching 


\section{INTRODUCCIÓN}

Estudiar las distintas maneras en que las personas perciben, analizan y estructuran la información para aprender, incluye múltiples aspectos que pueden aportar a la comprensión de los procesos de aprendizaje en los seres humanos (Morales-Ramírez et al., 2012; Romero et al., 2014; Shinnick y Woo, 2015). El aprendizaje no sólo debe entenderse como un conjunto de conocimientos que se desarrollan en la mente, sino también como habilidades que pueden modificarse y mejorarse con el tiempo (Alonso et al., 2012). Esto implica observar que hay diferentes preferencias o estilos (Gutiérrez et al., 2012; Abdul-Rahman y Boulay, 2014). Se tiene entonces, que los estilos de aprendizaje son rasgos cognitivos, afectivos y fisiológicos que funcionan como indicadores de cómo se percibe, se interrelaciona y responde a un ambiente de aprendizaje (Alonso et al., 2012; Espinosa y Estévez, 2013; Cakir et al., 2014). Estos rasgos se evidencian en la manera cómo los sujetos formalizan los esquemas de interpretación y su relación con los contenidos e información. Ventura et al., (2012) definen los estilos de aprendizaje como las tácticas con las cuales el ser humano asimila la información en su proceso cognitivo y cognoscitivo. Por su parte Ortíz y Canto, (2013) complementan esta definición añadiendo que, los estilos de aprendizaje se entienden como las variables personales, que a mitad de camino entre la inteligencia y la personalidad, explican las diferentes formas de abordar, planificar y responder ante las demandas del aprendizaje.

Los estilos de aprendizaje más comunes han sido sintetizados por Alonso et al., (2012) en el cuestionario Honey-Alonso Estilos de Aprendizaje (CHAEA). Dichos autores afirman que quienes manifiestan una preferencia por el estilo activo, suelen ser personas de mente abierta, nada escépticas, entusiasmadas por las tareas nuevas, animadores, improvisadores, descubridores, arriesgados, espontáneos (López-Aguado, 2011; Ventura et al., 2012; Espinosa y Estévez, 2013). Por su parte los reflexivos, tienden a ser analíticos, receptivos pacientes y prefieren observar con detenimiento antes de llegar a alguna conclusión final (Bahamón et al., 2013). Mientras que los individuos con prevalencia del estilo teórico, enfocan los problemas verticalmente y por etapas lógicas, adaptando e integrado las observaciones dentro de teorías metódicas, estructuradas y complejas (Bolívar-López y Rojas-Velásquez, 2013). Y los pragmáticos fundamentalmente suelen ser más realistas y prefieren la aplicación inmediata de las ideas aprendidas (Aguilera, 2012). Lo ideal, afirma Alonso et al., (2012) sería que todos los estudiantes fueran capaces de experimentar, reflexionar, elaborar hipótesis y aplicarlas en partes iguales; es decir, que todas las virtudes estuvieran repartidas equilibradamente. Sin embargo la experiencia indica que es difícil encontrar individuos con dichas características mentales (Morales-Ramírez et al., 2012). Safarin et al., (2013) señala que los estilos de aprendizaje serían la interiorización por parte de cada sujeto de una etapa determinada del ciclo.

Aunque los estudiantes compartan la experiencia de aprendizaje en las mismas aulas, cada uno tiene una personalidad diferente y su estructura de comportamiento ha sido fundamentada por las características innatas y de vivencias personales (Valenzuela y González, 2010). Algunas investigaciones como las realizadas por Abello et al., (2011) y Geçit y Delihasan (2014) indican que conocer el estilo de aprendizaje posibilita, mejorar la forma de enseñanza de los docentes. Autores como Tocci (2013) señalan que los estudiantes aprenden con más efectividad cuando se les enseña según sus estilos de aprendizaje predominantes.

Por otra parte, se dice que el género, también influye en la manera de aprender (Acevedo y Rocha, 2011; Baraz et al., 2014). López-Aguado (2011) infiere que los estudiantes de diferentes géneros podrían tener distintas tendencias en cuanto a su forma de aprender, desde el punto de vista de sus capacidades intelectuales y emocionales, de acuerdo a las características morfológicas y fisiológicas. Por ello, los docentes deben buscar la manera de crear ambientes propicios de aprendizaje, adaptando los procesos de enseñanza de acuerdo a las necesidades y características de cada estudiante (Sepúlveda-Carreño et al., 2011; Bahamón et al., 2013; y Eishani et al., 2014). De esta manera es indispensable que el docente conozca cómo los estudiantes interiorizan los conocimientos, especialmente en programas como los de Ingeniería, donde se requiere de una alta exigencia académica (Solís y Arcudia, 2010; Ventura, 2011; Tocci, 2013). El objetivo de este trabajo fue analizar los estilos de aprendizaje de los estudiantes de la Facultad de Ingeniería de la Universidad de Cartagena por medio del Cuestionario Honey-Alonso Estilos de Aprendizaje.

\section{MATERIALES Y MÉTODOS}

\section{Población y Muestra}

Para el desarrollo de este estudio se empleó un diseño metodológico descriptivo, no experimental, correlacional y de corte transversal, ya que las variables se midieron en un único momento temporal. Similar a lo realizado por Herrera y Zapata (2012) y Ortíz y Canto (2013). La población objeto de estudio estuvo conformada por todos los estudiantes de la Facultad de Ingenierías de la modalidad presencial en la Universidad de Cartagena, aproximadamente 1440 de todos los semestres. Entre los programas de 
Ingeniería de Alimentos, Ingeniería Química, Ingeniería Civil e Ingeniería de Sistemas. Se tomó una muestra aleatoria representativa del $10 \%$, equivalente a 144 estudiantes distribuida de forma equitativa, 72 mujeres (50\%) con una edad promedio de $22,31 \pm 0,39$ años y 72 hombres $(50 \%)$ de $21,92 \pm 0,43$ años.

\section{Instrumento de evaluación}

Para evaluar y medir los estilos de aprendizaje de los estudiantes de la Facultad de Ingenierías de la Universidad de Cartagena, se utilizó el Cuestionario Honey-Alonso de Estilos de Aprendizaje (CHAEA), debido a su amplia utilización en diversas investigaciones lberoamericanas; donde se resaltan estudios como el realizado por Bahamón et al., (2013). Dicho formato consta con un total de 80 enunciados, de los cuales 20 corresponden a cada estilo y se distribuyen aleatoriamente; Se usaron dos opciones: (1), si está de acuerdo, y (0), si está en desacuerdo. Esto permitió identificar los estilos básicos: activo, reflexivo, teórico y pragmático. Alonso et al., (2012) indicaron coeficientes de confiabilidad entre 0,58 y 0,72. Otros estudios recientes revelaron índices de confiabilidad alfa Cronbach del CHAEA entre 0,60 a 0,70 (Valenzuela y González, 2010; Morales et al., 2012; Ventura et al., 2012; Ortíz y Canto, 2013).

\section{Temporalización}

El presente estudio se realizó con estudiantes matriculados en el segundo semestre del 2014. Las pruebas se efectuaron en las instalaciones del Grupo de Investigación NUSCA (Nutrición, Salud y Calidad Alimentaria), del programa de Ingeniería de Alimentos de la Universidad de Cartagena, en forma de test en físico, con un límite promedio de 20 minutos, para cada estudiante.

\section{Análisis de datos}

Después de obtener la información se construyó una base de datos en el programa Excel 2013, para Windows y posteriormente en el Programa Estadístico para Ciencias Sociales, IBM SPSS ๔ - versión 19.0. Se introdujeron los perfiles de aprendizaje de los estudiantes y se analizaron los correspondientes porcentajes y promedios con sus desviaciones estándar. Para hallar las diferencias estadísticas entre los estilos de aprendizaje por género en los programas, se aplicó la prueba de t-Student y se realizó un análisis de varianza (ANOVA bifactorial) con un nivel de significancia del $5 \%(p \leq 0,05)$. Así mismo se realizó una correlación a partir de la prueba de Pearson, entre los estilos para estimar hasta qué nivel se relacionaron dichas variables. La correlación se consideró altamente significativa al nivel de 0,01 (bilateral). La preferencia del aprendizaje, se realizó teniendo en cuenta el Baremo de interpretación esquematizado en la Tabla 1, (Alonso et al., 2012).

Tabla 1: Baremo de preferencia estándar en los estilos de aprendizaje

\begin{tabular}{|c|c|c|c|c|c|}
\hline $\begin{array}{c}\text { Estilos de } \\
\text { Aprendizaje }\end{array}$ & $\begin{array}{c}10 \% \text { Muy } \\
\text { Baja }\end{array}$ & $20 \%$ Baja & $\begin{array}{c}40 \% \\
\text { Moderada }\end{array}$ & $20 \%$ Alta & $\begin{array}{c}10 \% \text { Muy } \\
\text { alta }\end{array}$ \\
\hline Activo & $0-6$ & $7-8$ & $9-12$ & $13-14$ & $15-20$ \\
\hline Reflexivo & $0-10$ & $11-13$ & $14-17$ & $18-19$ & 20 \\
\hline Teórico & $0-6$ & $7-9$ & $10-13$ & $14-15$ & $16-20$ \\
\hline Pragmático & $0-8$ & $9-10$ & $11-13$ & $14-15$ & $16-20$ \\
\hline
\end{tabular}

\section{RESULTADOS Y ANÁLISIS}

En la Tabla 2, se esquematizan los datos promedios de los estilos de aprendizajes obtenidos por géneros en cada uno de los programas de la Facultad de Ingenierías de la Universidad de Cartagena. Se puede notar que para todos los hombres el estilo predominante fue el reflexivo, con excepción de Ingeniería Civil, donde el teórico estuvo ligeramente por encima. Mientras que para las mujeres los datos fueron más variados, ya que en el caso de Ingeniería de Alimentos predominó el estilo teórico, en Civil el activo, en Sistema el reflexivo, y en Química el pragmático.

No hubo diferencias estadísticamente significativas entre hombres y mujeres $(p>0,05)$. Estos resultados coinciden con los reportados por López-Aguado (2011) quien no encontró diferencias significativas en los estilos de aprendizaje y género, de estudiantes universitarios. Así mismo Acevedo y Rocha (2011) identificaron los estilos de aprendizaje de alumnos de Ingeniería, y no encontraron diferencias significativas entre hombres y mujeres. 
Tabla 2: Datos promedios de los estilos de aprendizaje en la Facultad de Ingenierías

\begin{tabular}{|c|c|c|c|c|c|c|c|c|}
\hline Estilos & \multicolumn{2}{|c|}{ Activo } & \multicolumn{2}{|c|}{ Reflexivo } & \multicolumn{2}{|c|}{ Teórico } & \multicolumn{2}{|c|}{ Pragmático } \\
\hline Programa/Género & Hombres & Mujeres & Hombres & Mujeres & Hombres & Mujeres & Hombres & Mujeres \\
\hline Alimentos & $10,11 \pm 2,70$ & $11,39 \pm 4,68$ & $10,83 \pm 3,33$ & $11,72 \pm 5,44$ & $9,89 \pm 3,51$ & $12,06 \pm 4,49$ & $10,44 \pm 3,18$ & $11,61 \pm 4,34$ \\
\hline Civil & $10,44 \pm 4,83$ & $10,33 \pm 2,50$ & $12,02 \pm 5,27$ & $9,72 \pm 1,36$ & $12,78 \pm 4,11$ & $8,72 \pm 2,02$ & $11,33 \pm 4,86$ & $9,11 \pm 2,72$ \\
\hline Sistemas & $10,22 \pm 3,35$ & $9,78 \pm 3,17$ & $14,28 \pm 3,69$ & $16,39 \pm 2,55$ & $11,72 \pm 3,30$ & $13,22 \pm 3,44$ & $12,50 \pm 4,05$ & $12,01 \pm 4,18$ \\
\hline Química & $13,17 \pm 2,57$ & $10,51 \pm 3,09$ & $14,39 \pm 1,69$ & $11,61 \pm 4,47$ & $12,33 \pm 2,66$ & $10,78 \pm 4,01$ & $14,56 \pm 3,52$ & $12,29 \pm 3,71$ \\
\hline Estadístico & \multicolumn{2}{|c|}{$t=0,59$} & \multicolumn{2}{|c|}{$t=0,29$} & \multicolumn{2}{|c|}{$t=0,68$} & \multicolumn{2}{|c|}{$t=0,44$} \\
\hline p-valor & \multicolumn{2}{|c|}{$\mathrm{p}=0,12>0,05$} & \multicolumn{2}{|c|}{$\mathrm{p}=0,22>0,05$} & \multicolumn{2}{|c|}{$\mathrm{p}=0,26>0,05$} & \multicolumn{2}{|c|}{$\mathrm{p}=0,37>0,05$} \\
\hline
\end{tabular}

En el presente trabajo, la preferencia más alta del estilo reflexivo, estuvo en el programa Ingeniería Química para los hombres y en las mujeres del programa Ingeniería de Sistemas. En Ingeniería de Alimentos, las mujeres tuvieron puntajes más altos en todos los estilos evaluados, respecto a los hombres del mismo programa. En el caso de las demás Ingeniería esta diferencia no fue tan marcada. En la Tabla 3, se detallan los análisis de varianza bifactorial realizados en los perfiles de aprendizaje entre hombres y entre mujeres.

Tabla 3: Análisis de varianza bifactorial entre los perfiles de aprendizaje de Hombres y Mujeres

\begin{tabular}{|c|c|c|c|c|c|c|}
\hline \multicolumn{7}{|c|}{ Hombres (n=72) } \\
\hline Origen de las variaciones & $\begin{array}{c}\text { Suma de } \\
\text { cuadrados }\end{array}$ & $\begin{array}{c}\text { Grados de } \\
\text { libertad }\end{array}$ & $\begin{array}{c}\text { Cuadrados } \\
\text { medio }\end{array}$ & Valor F & $\begin{array}{c}\mathrm{p}- \\
\text { valor }\end{array}$ & $\begin{array}{c}\text { Crítico } \\
\mathrm{F}\end{array}$ \\
\hline Entre Programas (Bloques) & 22,14 & 3 & 7,38 & 3,76 & 0,07 & 3,86 \\
\hline $\begin{array}{c}\text { Estilos de Aprendizaje } \\
\text { (Grupos) }\end{array}$ & 7,51 & 3 & 2,52 & 2,97 & 0,09 & 3,86 \\
\hline Error & 7,58 & 9 & 0,84 & & & \\
\hline Total & 37,22 & 15 & & & & \\
\hline \multicolumn{7}{|c|}{ Mujeres ( $\mathrm{n}=72$ ) } \\
\hline $\begin{array}{c}\text { Origen de las variaciones } \\
\text { Suma de }\end{array}$ & $\begin{array}{c}\text { Grados de } \\
\text { cuadrados }\end{array}$ & $\begin{array}{c}\text { Cuadrados } \\
\text { libertad } \\
\text { medio }\end{array}$ & Valor F & $\begin{array}{c}\mathrm{p} \text { - } \\
\text { valor }\end{array}$ & $\begin{array}{c}\text { Crítico } \\
\mathrm{F}\end{array}$ \\
\hline $\begin{array}{c}\text { Entre Programas (Bloques) } \\
\text { Estilos de Aprendizaje } \\
\text { (Grupos) }\end{array}$ & 23,62 & 3 & 7,87 & 3,65 & 0,06 & 3,86 \\
\hline Error & 7,08 & 3 & 2,36 & 1,09 & 0,42 & 3,86 \\
\hline Total & 19,42 & 9 & 2,16 & & & \\
\hline
\end{tabular}

No se detectaron diferencias estadísticas en los estilos de aprendizaje entre los programas, tanto en hombres, como en mujeres ( $p>0,05)$. Lo que indica similitud en la forma de aprender de los estudiantes de los cuatro programas de la Facultad de Ingeniería de la Universidad de Cartagena. Estos resultados fueron similares a los hallados por Morales-Ramírez (2012) en estudiantes universitarios de Ingeniería Computacional e Informática Administrativa. Así mismo Solís y Arcudia (2010) no detectaron diferencias estadísticas en estudiantes de Ingeniería Civil.

La Figura 1, muestra la comparación global del perfil de aprendizaje entre hombres y mujeres. Se observaron promedios relativamente más altos en los hombres, especialmente en el estilo pragmático, lo que no fue significativo ( $p>0,05)$. Lo cual coincide con lo reportado por Sepúlveda-Carreño et al., (2011) en estudiantes de química y farmacia. Así como con lo hallado por Ortíz y Canto (2013), Espinosa y Estévez (2013) y Ventura et al., (2012) quienes investigaron los estilos de aprendizaje en estudiantes de Ingeniería y no encontraron diferencias estadísticas por género. Al contrastar los datos obtenidos de los estudiantes, se observó que predominó la preferencia moderada (33,33\%), lo que se esquematiza en la Tabla 4 . Esto indica que los estudiantes encuestados no poseían un nivel alto en los estilos analizados. Gutiérrez et al., (2012) y Romero et al., (2014), analizaron las variables que influyen en los estilos de aprendizaje de diferentes grupos de alumnos universitarios y encontraron variación en las preferencias del estilo activo en hombres, desde baja hasta muy alta, mientras que las mujeres presentaron preferencia moderada en los estilos reflexivo, teórico y pragmático. Morales-Ramírez (2012), Bolívar-López y Rojas-Velásquez (2013) informaron una preferencia alta en los estilos pragmático y activo por sobre teórico y reflexivo, en estudiantes universitarios. 


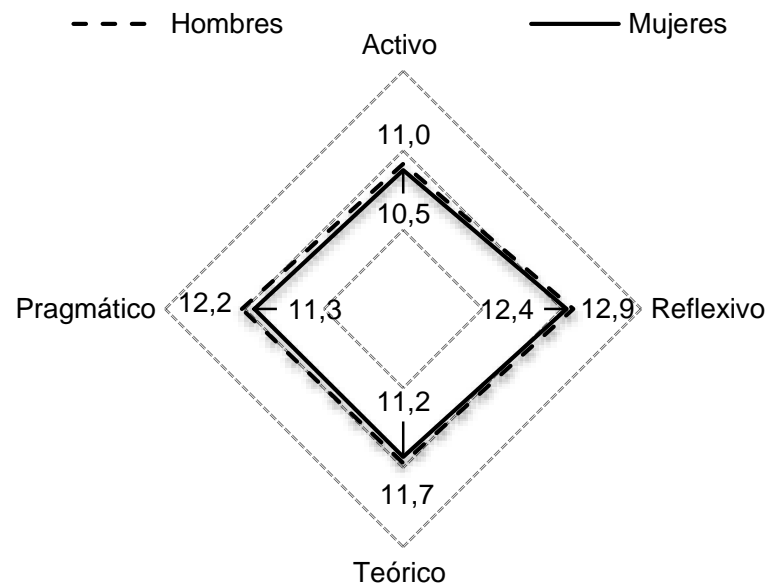

Fig.1: Perfil de los Estilos de Aprendizaje de los estudiantes encuestados

Tabla 4: Frecuencia preferencial de los estilos de aprendizaje en la muestra de estudiantes

\begin{tabular}{|c|c|c|c|c|c|c|c|c|c|}
\hline \multirow{3}{*}{$\begin{array}{c}\text { Preferencia/ } \\
\text { Género }\end{array}$} & \multicolumn{9}{|c|}{ Estilos de Aprendizaje } \\
\hline & \multicolumn{2}{|c|}{ Activo } & \multicolumn{2}{|c|}{ Reflexivo } & \multicolumn{2}{|c|}{ Teórico } & \multicolumn{2}{|c|}{ Pragmático } & \multirow{2}{*}{ Total (\%) } \\
\hline & Hombre & Mujer & Hombre & Mujer & Hombre & Mujer & Hombre & Mujer & \\
\hline Muy Baja & $\begin{array}{c}12 \\
(2,1 \%)\end{array}$ & $\begin{array}{c}10 \\
(1,7 \%)\end{array}$ & $\begin{array}{c}20 \\
(3,5 \%)\end{array}$ & $\begin{array}{c}32 \\
(5,6 \%)\end{array}$ & $7(1,2 \%)$ & $\begin{array}{c}13 \\
(2,3 \%)\end{array}$ & $\begin{array}{c}15 \\
(2,6 \%)\end{array}$ & $\begin{array}{c}19 \\
(3,3 \%)\end{array}$ & $\begin{array}{c}128 \\
(22,22)\end{array}$ \\
\hline Baja & $7(1,2 \%)$ & $6(1,1 \%)$ & $\begin{array}{c}18 \\
(3,1 \%)\end{array}$ & $\begin{array}{c}10 \\
(1,7 \%)\end{array}$ & $\begin{array}{c}18 \\
(3,1 \%)\end{array}$ & $\begin{array}{c}13 \\
(2,3 \%)\end{array}$ & $4(0,7 \%)$ & $9(1,6 \%)$ & $\begin{array}{c}85 \\
(14,81)\end{array}$ \\
\hline Moderada & $\begin{array}{c}26 \\
(4,5 \%)\end{array}$ & $\begin{array}{c}35 \\
(6,1 \%)\end{array}$ & $\begin{array}{c}23 \\
(4,0 \%)\end{array}$ & $\begin{array}{c}14 \\
(2,4 \%)\end{array}$ & $\begin{array}{c}23 \\
(4,0 \%)\end{array}$ & $\begin{array}{c}23 \\
(4,0 \%)\end{array}$ & $\begin{array}{c}24 \\
(4,2 \%)\end{array}$ & $\begin{array}{c}24 \\
(4,2 \%)\end{array}$ & $\begin{array}{c}192 \\
(33,33)\end{array}$ \\
\hline Alta & $\begin{array}{c}13 \\
(2,3 \%) \\
\end{array}$ & $\begin{array}{c}15 \\
(2,6 \%) \\
\end{array}$ & $\begin{array}{c}11 \\
(1,9 \%)\end{array}$ & $\begin{array}{c}14 \\
(2,4 \%) \\
\end{array}$ & $\begin{array}{c}11 \\
(1,9 \%) \\
\end{array}$ & $\begin{array}{c}13 \\
(2,3 \%) \\
\end{array}$ & $\begin{array}{c}10 \\
(1,7 \%) \\
\end{array}$ & $\begin{array}{c}11 \\
(1,9 \%) \\
\end{array}$ & $\begin{array}{c}98 \\
(17,22) \\
\end{array}$ \\
\hline Muy Alta & $\begin{array}{c}14 \\
(2,4 \%)\end{array}$ & $6(1,0 \%)$ & $0(0,0 \%)$ & $2(0,4 \%)$ & $\begin{array}{c}13 \\
(2,3 \%)\end{array}$ & $\begin{array}{c}10 \\
(1,7 \%)\end{array}$ & $\begin{array}{c}19 \\
(3,3 \%)\end{array}$ & $9(1,6 \%)$ & $\begin{array}{c}73 \\
(12,72)\end{array}$ \\
\hline
\end{tabular}

Estos resultados coinciden con los reportados por Laugero et al., (2009), Aguilera (2012), Bahamón et al., (2013) y Ortíz y Canto, (2013) quienes analizaron los estilos de aprendizaje en estudiantes universitarios y encontraron tendencias hacia la preferencia moderada. Por su parte Ventura et al., (2012) señalaron preferencias de aprendizaje moderadas en los estilos activo y teórico, de estudiantes universitarios. Valenzuela y González, (2010) hallaron prevalencia moderada en el estilo reflexivo de estudiantes de Contabilidad, de activo-moderado en estudiantes de derecho y pragmático-alta, en estudiantes de comunicación. En la Tabla 5, se esquematiza detalladamente la distribución de los perfiles de aprendizajes por género entre los cuatro programas de la Facultad de Ingeniería.

Tabla 5: Perfiles de aprendizajes en la Facultad de Ingeniería. Activo (A), Reflexivo (R), Teórico (T), Pragmático (P)

\begin{tabular}{|c|c|c|c|c|c|c|c|c|c|c|}
\hline Programas & \multicolumn{2}{|c|}{$\begin{array}{c}\text { Ingeniería } \\
\text { Alimentos }\end{array}$} & \multicolumn{2}{|c|}{ Ingeniería Civil } & \multicolumn{2}{c|}{$\begin{array}{c}\text { Ingeniería } \\
\text { Sistemas }\end{array}$} & \multicolumn{2}{c|}{$\begin{array}{c}\text { Ingeniería } \\
\text { Química }\end{array}$} & \multicolumn{2}{c|}{ Total } \\
\hline $\begin{array}{c}\text { Estilos/Gén } \\
\text { eros }\end{array}$ & $\begin{array}{c}\text { Hombre } \\
\mathrm{s}(\mathrm{n}=18)\end{array}$ & $\begin{array}{c}\text { Mujeres } \\
(\mathrm{n}=18)\end{array}$ & $\begin{array}{c}\text { Hombre } \\
\mathrm{s}(\mathrm{n}=18)\end{array}$ & $\begin{array}{c}\text { Mujeres } \\
(\mathrm{n}=18)\end{array}$ & $\begin{array}{c}\text { Hombre } \\
\mathrm{s}(\mathrm{n}=18)\end{array}$ & $\begin{array}{c}\text { Mujeres } \\
(\mathrm{n}=18)\end{array}$ & $\begin{array}{c}\text { Hombre } \\
\mathrm{s}(\mathrm{n}=18)\end{array}$ & $\begin{array}{c}\text { Mujere } \\
\mathrm{s} \\
(\mathrm{n}=18)\end{array}$ & $\begin{array}{c}\text { Hombre } \\
\mathrm{s}(\mathrm{n}=72)\end{array}$ & $\begin{array}{c}\text { Mujeres } \\
(\mathrm{n}=72)\end{array}$ \\
\hline $\mathrm{A}$ & 22,22 & 16,67 & 16,67 & 33,33 & 11,11 & 5,55 & 16,65 & - & 16,67 & 13,89 \\
\hline $\mathrm{R}$ & 22,22 & 33,33 & 27,79 & 22,22 & 72,22 & 66,67 & 11,11 & 22,22 & 31,94 & 36,11 \\
\hline $\mathrm{T}$ & 11,11 & 16,67 & 16,59 & - & - & 9,44 & 5,56 & 38,89 & 8,33 & 16,67 \\
\hline $\mathrm{P}$ & 11,11 & 22,22 & 22,32 & 11,11 & 5,56 & 12,78 & 44,44 & 33,33 & 20,83 & 19,44 \\
\hline $\mathrm{A}-\mathrm{R}$ & - & - & 5,52 & 22,22 & 11,11 & - & 5,56 & - & 4,17 & 5,56 \\
\hline $\mathrm{R}-\mathrm{T}$ & 11,11 & 11,11 & - & - & - & - & 5,56 & - & 4,17 & 2,78 \\
\hline $\mathrm{R}-\mathrm{P}$ & 5,56 & - & - & 5,56 & - & 5,56 & 5,56 & - & 5,56 & 2,78 \\
\hline $\mathrm{T}-\mathrm{P}$ & 16,67 & - & 11,11 & 5,56 & - & - & 5,56 & 5,56 & 8,33 & 2,78 \\
\hline Total & 100 & 100 & 100 & 100 & 100 & 100 & 100 & 100 & 100 & 100 \\
\hline
\end{tabular}


Se observó que en Ingeniería de Alimentos el 44,44\% de los hombres encuestados, indicó que su estilo de aprendizaje estuvo entre activo y reflexivo; y el 16,67\% entre teórico y pragmático; mientras que para las mujeres los estilos más frecuentes fueron reflexivo y pragmático con un 55,55\%. En los hombres de Ingeniería Civil el 50,11\% estuvo entre reflexivo y pragmático. Y en las mujeres el $77,77 \%$ estuvo entre activo y reflexivo. En el programa de Ingeniería de Sistemas se observó que los estilos de aprendizajes predominantes en los hombres fueron el reflexivo y el activo $(94,44 \%)$, mientras que para las mujeres fueron el reflexivo y el pragmático (85,01\%). En el programa Ingeniería Química los hombres indicaron una marcada preferencia por los estilos activo y pragmático $(61,09 \%)$, así mismo se observó preferencia multimodal. Y en las mujeres los mayores porcentajes estuvieron entre teórico y el pragmático (77,78\%). En general los estilos predominantes tanto en hombres como en mujeres fueron el reflexivo y el pragmático, lo cual indica que los estudiantes encuestados de la Facultad de Ingeniería de la Universidad de Cartagena tienden a ser personas analíticas, receptivas, con preferencia por el trabajo experimental.

Según Alonso et al., (2012) y Shinnick y Woo (2015) los alumnos reflexivos aprenden mejor cuando pueden observar detenidamente, trabajar a su propio ritmo, investigar y hacer análisis detallados. Por el contrario a estos estudiantes se les dificulta el aprendizaje cuando no tienen datos suficientes para procesar información y cuando les toca hacer trabajos de forma superficial (Sepúlveda-Carreño et al., 2011; Waes et al., 2014). Mientras que los estudiantes pragmáticos aprenden mejor cuando, tienen la posibilidad inmediata de aplicar lo aprendido, de experimentar, elaborar planes de acción con resultados evidentes, dar indicaciones, y tratar con expertos que son capaces de hacer las cosas por ellos mismos (Bahamón et al., 2013; Giuliano et al., 2014). Y se les hace difícil el aprendizaje cuando perciben que lo aprendido no tiene ninguna aplicación práctica ante la realidad. Estos resultados respaldan en cierta medida los hallazgos de Safarin et al., (2013) quienes comunicaron una tendencia hacia el perfil reflexivo de estudiantes de Malasia, inscritos en carreras con componentes experimentales y empírico-analíticos como las de Ingeniería. Lo anterior también coincide con lo encontrado por Solís y Arcudia (2010) según el cual el punto fuerte de los estudiantes de Ingeniería, es probablemente la capacidad de asimilar gran cantidad de información, abstraer los conceptos y patrones generales aplicándolos de manera objetiva, es decir, muestran una tendencia más reflexiva - pragmática.

Ortíz y Canto, (2013) encontraron predominio del estilo pragmático, en estudiantes de Ingeniería en México. Lo que sugiere que los jóvenes universitarios de Ingeniería, suelen ser más minuciosos y pacientes que otros estudiantes de pregrado. Por su parte Laugero et al., (2009), encontraron que el estilo de aprendizaje predominante fue el reflexivo en estudiantes mexicanos de Ingeniería Mecánica, Eléctrica, Metalurgia, Industrial y Electrónica. Safarin et al., (2013) indicó que los estilos reflexivo y pragmático, se adaptan al perfil profesional de los estudiantes de Ingeniería, por lo que sugiere que las metodologías de enseñanza deben ir en armonía con dicho estilo predominante. Ya que por su naturaleza la Ingeniería exige un análisis detallado de los fenómenos reales y demanda a la vez soluciones rápidas de diversas problemáticas. Los datos recogidos con el CHAEA en la Facultad de Ingeniería de la Universidad de Cartagena, mostraron que una parte importante de los estudiantes hacen uso de varios estilos de aprendizaje, lo cual sugiere cierta multimodalidad en el desempeño académico cuando realizan procesos para adquirir nuevos conocimientos y aprenderlos. Lo cual coincide con lo hallado por Gutiérrez et al., (2012) y Bahamón et al., (2013), quienes también encontraron estilos de aprendizaje multimodales en estudiantes universitarios.

Ventura (2011) encontró uso de estilos multimodales en estudiantes Universitarios y aseveró que si bien es cierto que los estilos de aprendizaje son esquemas de comportamiento, estos no suelen ser fijos, predeterminados, si no que por el contrario, pueden cambiar según la situación de aprendizaje y experiencia que va adquiriendo el aprendiz. En cuanto a la transformación de éstos, las investigaciones como las de Laugero et al., (2009), Acevedo y Rocha (2011), Abdul-Rahman y Boulay (2014) y Romero et al., (2014) indicaron que el estudiante modifica sus estilos de aprendizaje en la medida en que avanza en sus estudios y grados académico, lo cual podría evidenciar un proceso de adaptación cognitiva a la vida universitaria y a las estrategias pedagógicas empleadas por los educadores. De acuerdo con esto, es importante que al estudiante se le enseñe a identificar su propio estilo para movilizar un aprendizaje más eficiente y efectivo (Abello et al., 2011).

\section{Correlación entre los estilos de aprendizaje}

En la Tabla 6, se enmarcan las correlaciones entre cada estilo de aprendizaje de los estudiantes por géneros. En la parte superior se muestran los datos de los hombres, en los cuales se puede ver que no hubo ninguna relación entre los estilos reflexivo y el pragmático con el activo $(p>0,05)$. Mientras que en los estilos teórico y activo se observó una relación lineal y significativa $(r=0,27$ y $p<0,05)$. Así mismo entre el reflexivo con el teórico $(r=0,36$ y $p=0,01)$ y el pragmático $(r=0,37$ y $p<0,01)$ la relación fue altamente significativa; al igual que entre el teórico y el pragmático $(r=0,38$ y $p<0,01)$. Resultados que coinciden con los reportados por Herrera y Zapata (2012). Así mismo Gutiérrez et al., (2012) y Tocci (2013) indicó una correlación positiva y significativa entre el estilo teórico y el activo. 
Tabla 6: Correlación entre estilos de aprendizaje de los estudiantes de la Facultad de Ingeniería por género. ${ }^{*}$ Correlación: significativa a 0,05 (bilateral), $y$ **altamente significativa a 0,01 (bilateral).

\begin{tabular}{|c|c|c|c|c|c|c|}
\hline \multirow{2}{*}{\multicolumn{3}{|c|}{ Estilos de Aprendizaje }} & \multicolumn{4}{|c|}{ Hombres $(n=72)$} \\
\hline & & & \multirow[t]{2}{*}{ Activo } & \multirow{2}{*}{$\frac{\text { Reflexivo }}{0,16}$} & \multirow{2}{*}{$\begin{array}{r}\text { Teórico } \\
0,27^{\star}\end{array}$} & \multirow{2}{*}{$\begin{array}{r}\text { Pragmático } \\
0,20\end{array}$} \\
\hline \multirow{8}{*}{ 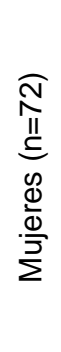 } & \multirow{2}{*}{ Activo } & Coeficiente de Pearson & & & & \\
\hline & & $\mathrm{p}$-valor & & 0,19 & 0,02 & 0,09 \\
\hline & \multirow{2}{*}{ Reflexivo } & Coeficiente de Pearson & $-0,09$ & 1 & $0,36^{* *}$ & $0,37^{* *}$ \\
\hline & & $\mathrm{p}$-valor & 0,47 & & 0,00 & 0,00 \\
\hline & \multirow{2}{*}{ Teórico } & Coeficiente de Pearson & $-0,28$ & $0,44^{\star *}$ & 1 & $0,38^{* *}$ \\
\hline & & $\mathrm{p}$-valor & 0,05 & 0,00 & & 0,00 \\
\hline & \multirow{2}{*}{ Pragmático } & Coeficiente de Pearson & $0,33^{* *}$ & $0,24^{*}$ & $-0,06$ & 1 \\
\hline & & p-valor & 0,00 & 0,04 & 0,63 & \\
\hline
\end{tabular}

Al analizar los datos de las mujeres esquematizados en la parte inferior, se observó una relación directa y altamente significativa entre el estilo activo y pragmático $(r=0,33$ y $p<0,01)$. Al igual que el reflexivo con el teórico $(r=0,44$ y $p<0,01)$ y el pragmático $(r=0,24$ y $p<0,05)$. Mientras que entre el activo y el teórico se observó una correlación inversa y significativa $(r=-0,28$ y $p<0,05)$. En los estilos activo y reflexivo y entre pragmático y teórico se encontró una relación inversa, pero no significativa $(p>0,05)$. Resultados similares fueron reportados por López-Aguado (2011), Sepúlveda-Carreño et al., (2011), Ortíz y Canto (2013) y Eishani et al., (2014) en estudiantes universitarios.

En general se necesita más investigación para comparar y correlacionar los estilos de aprendizaje de los estudiantes, con los docentes de las Facultades de Ingeniería. La identificación del estilo de aprendizaje predominante de los estudiantes de Ingeniería puede ser una herramienta útil para adaptar la metodología de enseñanza de los docentes.

\section{CONCLUSIONES}

El estilo de aprendizaje predominante, al analizar los resultados obtenidos en la Facultad de Ingenierías de la Universidad de Cartagena fue el reflexivo, seguido por el pragmático, el activo y por último el teórico, lo que permite considerar que los estudiantes de los cuatro programas de Ingeniería tienden a ser receptivos, analíticos, y prefieren la aplicación de las ideas aprendidas en las aulas de clase, lo que implica una valiosa información para los docentes, que deben tener en cuenta esto al momento de diseñar los métodos de enseñanza. No se observaron diferencias estadísticamente significativas por género $(p>0,05)$ en cada uno de los estilos analizados. La preferencia general para cada estilo estuvo enmarcada en el nivel de moderado. En los hombres se observó correlación positiva y significativa entre los estilos activo y teórico $(p<0,05)$, así como en el reflexivo con el pragmático $(p<0,01)$. Mientras que en las mujeres se observó una relación directa y altamente significativa entre el estilo activo con el pragmático $(p<0,01)$. Así mismo en estas se presentó una alta correlación entre el estilo reflexivo y con el teórico $(p<0,01)$. Mientras que entre el activo y el teórico se observó una correlación inversa y significativa $(p<0,05)$.

\section{REFERENCIAS}

Abdul-Rahman, S. y B. Boulay, Learning programming vía worked-examples: Relation of learning styles to cognitive load, Computers in Human Behavior: 30 (1) 286-298 (2014).

Abello, D., C. Hernández y C. Hederich, Estilos de enseñanza en docentes universitarios, propuesta y validación de un modelo teórico e instrumental, Pedagogía y Saberes: 34(2), 141-154 (2011).

Acevedo, C. y F. Rocha, Estilos de Aprendizaje, género y rendimiento Académico, Revista Estilos de Aprendizaje: 8(8), 71-84 (2011).

Aguilera, P.E, Los estilos de enseñanza, una necesidad para la atención de los estilos de aprendizaje en la educación universitaria, Revista Estilos de Aprendizaje: 10 (1), 79-87 (2012).

Alonso, C., D. Gallego, y P. Honey, Los estilos de aprendizaje Procedimientos de diagnóstico y mejora (Octava ed.). Bilbao: Mensajero (2012).

Bahamón, M., M. Vianchá, L. Alarcón y C. Bohórquez, Estilos y estrategias de aprendizaje relacionadas con el logro académico en estudiantes universitarios, Pensamiento Psicológico: 11(1), 115-129 (2013).

Baraz, S., R. Memarian, y Z. Vanak, The diversity of Iranian nursing students' clinical learning styles: A qualitative study, Nurse Education in Practice: 14(5) 525-531 (2014). 
Bolívar-López, M. y F. Rojas-Velásquez, Los estilos de aprendizaje y el locus de control en estudiantes que inician estudios superiores y su vinculación con el rendimiento académico, Investigación y Postgrado: 23(3) 199-215 (2013).

Cakir, S. y otros tres autores, The Survey of Academic Procrastionation on High School Students with in Terms of School Burn-out and Learning Styles, Procedia - Social and Behavioral Sciences: 114, 654-662 (2014).

Eishani, K., E. Saa'd, y Y. Nami, The Relationship Between Learning Styles And Creativity, Procedia - Social and Behavioral Sciences: 114 (1) 52 - 55 (2014).

Espinosa, C. y G. Estévez, Auto-conocimiento de los estilos de aprendizaje, aspecto esencial en la actividad de estudio, Revista Iberoamericana sobre Calidad, Eficacia y Cambio en Educación: 11(3) 123-137 (2013).

Geçit, Y. y S. Delihasan, Learning Styles of Candidates of Geography Teaching, Procedia - Social and Behavioral Sciences, 120 (2) 621-628 (2014).

Giuliano, C. y cinco autores, Use of a unified learning style model in pharmacy curricula, Currents in Pharmacy Teaching and Learning: 6(1) 41-57 (2014).

Gutiérrez, M., J. García y D. Vieira, Estudio de las variables que influyen en los estilos de aprendizaje de diferentes grupos de alumnos de la Universidad de Valladolid, España, Revista Estilos de Aprendizaje: 10 (10), 55-64 (2012).

Herrera, C. y C. Zapata, Estudio correlacional de estilos de aprendizaje de estudiantes con modalidad en ciencias naturales, Tecné, Episteme y Didaxis: 31, 27-43 (2012).

Laugero, L., G. Balcaza y N. Salinas, "Una indagación en el Estilo de Aprendizaje de los Alumnos en Distintos Momentos de su Vida Universitaria", Revista Estilos de Aprendizaje: 4 (4), 1-13 (2009).

López-Aguado, M. Estilos de aprendizaje, Diferencias por género, curso y titulación, Revista Estilos de Aprendizaje: 7 (7), 109-134 (2011).

Morales-Ramírez, A, y cuatro autores, "Estilos de aprendizaje en estudiantes universitarios de Ingeniería en Computación e Informática Administrativa”, Revista Estilos de Aprendizaje: 9 (9), 156-168 (2012).

Ortíz, A. y P. Canto, Estilos de aprendizaje y rendimiento académico en estudiantes de Ingeniería en México, Revista Estilos de Aprendizaje: 11(11), 1-12 (2013).

Romero, L., M. Alarcón, y M. Gómez, Learning styles and web technology use in Business and Economics university students Carlota, Procedia - Social and Behavioral Sciences: 141 (2) 1281 - 1290 (2014).

Safarin, M., N. Mohd., K. Subari y M. Zolkiflibin, Visualization Skills and Learning Style Patterns among Engineering Students at Universiti Teknologi Malaysia, Procedia - Social and Behavioral Sciences: 93(2) 1769-1775 (2013).

Sepúlveda-Carreño, M.J, y otros cinco autores, Diferencias de género en el rendimiento académico y en el perfil de estilos y de estrategias de aprendizaje en estudiantes de química y farmacia de la Universidad de Concepción, Revista Estilos de Aprendizaje: 7(7), 1-19 (2011).

Shinnick, M.A y Woo, M, Learning style impact on knowledge gains in human patient simulation, Nurse Education Today: 35 (1) 63-67 (2015).

Solís, R. y C. Arcudia, Estilos de aprendizaje de los estudiantes de ingeniería civil, Revista Educación en Ingeniería: 10 (1), 24-36 (2010).

Tocci, A. Estilos de aprendizaje de los alumnos de ingeniería según la programación neuro lingüística, Revista estilos de aprendizaje: 11(12), 12-20 (2013).

Valenzuela, A. y V. González. "Estilos de aprendizaje de los estudiantes de la Universidad de Sonora, México. Estudio de caso", Revista de Estilos de Aprendizaje: 6 (6) 92-102 (2010).

Ventura, A., N. Moscoloni y R. Gagliardi, Estudio comparativo sobre los estilos de aprendizaje de estudiantes universitarios argentinos de diferentes disciplinas, Psicología desde el Caribe: 29(2) 276-304 (2012).

Ventura, A.C. Estilos de aprendizaje y prácticas de enseñanza en la universidad. Un binomio que sustenta la calidad educativa, Perfiles Educativos: 33(Esp.) 142-154 (2011).

Waes, L., D. Weijen y M. Leijen, Learning to write in an online writing center: The effect of learning styles on the writing process, Computers \& Education: 73(1), 60-71 (2014). 